\title{
Studies on the Group F Antigen of Lactobacilli: Isolation of a Teichoic Acid-Lipid Complex from Lactobacillus fermenti NCTC 6991
}

\author{
By A. J. WICKEN \\ School of Microbiology, University of New South Wales, P.O. Box I, \\ Kensington, N.S.W. 2033, Australia \\ AND K. W. KNOX \\ Institute of Dental Research, United Dental Hospital, Surry Hills, \\ N.S.W. 20Io, Australia
}

(Accepted for publication II November 1969)

SUMMARY

Membrane glycerol teichoic acid has been extracted from Lactobacillus fermenti NCTC $699 \mathrm{I}$ by two different procedures. Phenol extraction gave a complex of teichoic acid with glycolipid and phospholipid (lipoteichoic acid). Trichloracetic acid extraction resulted in degraded lipoteichoic acid and free teichoic acid chains. The teichoic acid is a $\mathrm{I}: 3$ phosphodiester linked glycerophosphate polymer substituted with D-alanine, D-galactose and a disaccharide of D-galactose and D-glucose. The significance of lipoteichoic acid as a membrane constituent is discussed.

\section{INTRODUCTION}

Glycerol teichoic acids occur as intracellular components in probably all Grampositive bacteria. They may be isolated by extraction, with trichloracetic acid (Critchley, Archibald \& Baddiley, 1962) or phenol (Burger \& Glaser, 1964), of the gelatinous pellet of ribosomes and membrane fragments obtained by high-speed centrifugation of wall-free disrupted organisms. The occurrence of glycerol teichoic acids as contaminants in preparations of bacterial ribosomes (Wicken \& Baddiley, I963) and DNA (Young \& Jackson, 1966) has been noted previously. A number of investigators (Hay, Wicken \& Baddiley, I963; Shattock \& Smith, I963; Shockman \& Slade, I964; Smith \& Shattock 1964) have suggested that the glycerol teichoic acid which is antigenically specific for group D Streptococcus faecalis is located in or on the external surface of the protoplast membrane. These findings have led to intracellular teichoic acids being generally regarded as membrane teichoic acids (Archibald, Baddiley \& Blumsom, 1968) although the chemical nature of this association remains undefined. The serological activity of membrane teichoic acids from a number of Gram-positive bacteria in precipitin tests with sera prepared against whole organisms has been well established. Purified teichoic acids have not been shown to act as antigens unless they are first made particulate by complexing with cationic precipitating agents (Burger, I966).

Sharpe, Davison \& Baddiley (I964) suggested that the serological activity of group F 
lactobacilli (e.g. Lactobacillus fermenti) might reside in an intracellular (membrane) teichoic acid although no analytical data were reported. In experiments designed to confirm this supposition we have isolated the membrane teichoic acid from $L$. fermenti NCTC 699I as a complex with lipid in a form which retains antigenicity and will sensitize normal sheep red blood cells. This paper describes the isolation procedure and a partial characterization of the complex while following papers (Hewett, Knox \& Wicken, 1970; Knox, Hewett \& Wicken, 1970) describe the serological properties of this complex.

\section{METHODS}

Organism. Lactobacillus fermenti NCTC 699I was originally obtained from the National Collection of Type Cultures, Colindale, London; this strain was employed by Sharpe (1955) in the serological classification of lactobacilli and belongs to serological group F (Sharpe \& Wheater, 1957).

Preparation of teichoic acid extracts. Organisms were grown and disrupted as described previously (Knox \& Holmwood, I968). Cell walls were removed by centrifugation at $12,000 \mathrm{~g}$ for $20 \mathrm{~min}$. (Servall $\mathrm{RC}_{2}$ ), and the non-sedimentable material retained as a source of membrane teichoic acids. Centrifugation of this material at $100,000 \mathrm{~g}$ (Spinco L 2-65) yielded a gelatinous pellet and a clear supernatant fraction, both of which were used as a source of teichoic acid. These fractions were freeze-dried and then reconstituted in water $3 \%(\mathrm{w} / \mathrm{v})$ for the gel fraction and $10 \%(\mathrm{w} / \mathrm{v})$ for the supernatant fraction). Two procedures were utilized for extracting teichoic acid from these fractions. (i) The crude material was stirred with an equal volume of $20 \%$ trichloracetic acid (TCA) at $4^{\circ}$ for $24 \mathrm{hr}$. Insoluble material was removed by centrifugation and re-extracted for $24 \mathrm{hr}$ with a further volume of $10 \%$ TCA. The combined extracts were washed repeatedly with ether to remove TCA and then teichoic acid was recovered from the aqueous layer by freeze-drying. (ii) The crude material was shaken with an equal volume of $90 \%$ aqueous phenol at 4 for I hr. After centrifugation, the aqueous layer was removed and the phenol layer washed with one volume of water. The combined aqueous layers were dialysed against three changes of distilled water and the non-diffusible material concentrated by rotary evaporation and finally freeze dried. The dried material was stirred with chloroform + methanol $(2+\mathrm{I}, \mathrm{v} / \mathrm{v})$ for $2 \mathrm{hr}$ at room temperature and insoluble material recovered by filtration through sintered glass. Nucleic acids in this preparation were degraded by incubation under toluene with deoxyribonuclease and ribonuclease (Sigma Chemical Co.) in $0.05 \mathrm{M}$-tris buffer, $\mathrm{pH} 8.0$, for $\mathrm{I} 6 \mathrm{hr}$ at $37^{\circ}$ (2 mg. of each enzyme + I m-mole $\mathrm{MgCl}_{2} / 100 \mathrm{ml}$. buffer). The digest was extracted with phenol as described above and the aqueous fraction dialysed against distilled water. Teichoic acid was recovered by freeze drying and extracted once more with chloroform + methanol and finally dried in vacuo.

Gel chromatography. Gel chromatography was routinely carried out on columns of Sephadex $\mathrm{G}_{75}$ and (G200 (Pharmacia) and 6\% agarose (Litex, Denmark) at ambient temperatures with $0.2 \mathrm{M}$ ammonium acetate, $\mathrm{pH} 6 \cdot 9$, as elutant. Fractions (about $5 \mathrm{ml}$.) were collected automatically by drop-counting using standard LKB equipment and samples were analysed for organic phosphorus and extinction at $260 \mathrm{~m} \mu$. Column void volumes were determined by the elution volumes of blue dextran (Pharmacia).

Analytical methods. Conditions for the acidic and alkaline hydrolysis of teichoic acids, enzymic dephosphorylation, characterization of glycosides and the identifica- 
tion of products by paper chromatography, unless stated in the text, are essentially as described previously (Forrester \& Wicken, I966; Wicken, 1966). Glucose was determined by the glucose oxidase method of Huggett \& Nixon (I957), galactose by the galactostat reagent (Worthington) and phosphorus by the method of Ames (I 966). Fatty acid esters were determined by a micro-modification of the method of Snyder \& Stephens (1959). Gas liquid chromatography of fatty acids was kindly performed by Dr M. Birmingham, Department of Biochemistry, University of Sydney.

Paper and thin-layer chromatography. The following solvent systems were used for paper chromatography: (A) propan-I-ol + aq. ammonia (sp.gr. o.88) + water $(6+3+\mathrm{I}$ by vol., Hanes \& Isherwood, I949), Whatman paper no. 4, ascending; (B) butan-I-ol + pyridine + water $(6+4+3$, by vol., Jeanes, Wise \& Dimler, 195I), Whatman paper, nos. I or $3 \mathrm{MM}$, descending; (C) ethyl acetate + pyridine + water $(5+2+5$ by vol., upper layer, Sastry \& Kates, 1964). Whatman paper no. I or $3 \mathrm{MM}$, descending. For thin layer chromatography, silica gel $G$ plates (E. Merck A-G) were prepared and activated at $105^{\circ}$ for $\mathrm{I} 6 \mathrm{hr}$ before use. Plates were developed in either (D), chloroform + methanol + water $\left(65+25+4\right.$, by vol.) or (E) light petroleum (b.p. 40 to $\left.60^{\circ}\right)+$ ethyl ether + acetic acid $(80+20+\mathrm{I}$, by vol. $)$.

The following spray reagents were used where applicable: periodate + Schiff's reagent for polyols and glycosides (Baddiley, Buchanan, Handschumacher \& Prescott, I956); perchloric acid + molybdate reagent for phosphates (Hanes \& Isherwood, 1949); alkaline silver nitrate (Trevelyan, Procter \& Harrison, I950) for reducing compounds and modified according to Brundish, Shaw \& Baddiley, (1965) for glycosides; ninhydrin for amino acids (Consden \& Gordon, 1948); rhodamine G6 (Dittmer \& Lester, 1964) and iodine vapour for lipids; dichlorofluorescein + aluminium chloride + ferric chloride (Dudzinski, 1967) for fatty acids.

Isolation of glycolipid. Lipid was extracted from freeze-dried whole organisms with chloroform + methanol $(2+\mathrm{I}, \mathrm{v} / \mathrm{v})$ as described by Brundish, Shaw \& Baddiley ( 1965$)$. Non-lipid contaminants were removed with Sephadex G25 (Wells \& Dittmer I963). A portion of the purified lipid (I00 mg.) in chloroform + methanol $(9+\mathrm{I}, \mathrm{v} / \mathrm{v})$ was applied to a column of silicic acid ( 10 g., Sigma SIL-LC) and eluted successively with $75 \mathrm{ml}$. amounts of chloroform, acetone and methanol. Thin-layer chromatography showed the bulk of the glycolipid to be present in the acetone fraction. Glycolipids in this fraction were deacylated (Wilkinson, 1968) without further purification and the constituent glycosides separated by paper chromatography in solvent B.

\section{RESULTS}

\section{Gel chromatography of teichoic acid extracts}

Phenol-extracted teichoic acids were excluded from columns of Sephadex $\mathrm{G}_{75}$ and G200 but were included by $6 \%$ agarose columns being eluted as single peaks close to the void volume. Identical elution volumes for teichoic acid were obtained for material extracted from gel or supernatant fractions (see Methods) and contaminating high molecular weight nucleic acid material did not occur in the same peak as teichoic acid. Fractions containing teichoic acid were combined, dialysed and freeze dried. Such preparations were designated as P-teichoic acids with the notations (gel) and (sol), where necessary, indicating origin from gel or supernatant fractions respectively. An elution diagram of P-teichoic acid (gel) from $6 \%$ agarose is shown in Fig. I. 
Dr M. W. A. Thomas, Department of Biochemistry, University of Sydney, kindly performed an ultracentrifugal analysis of $\mathrm{I} \%(\mathrm{w} / \mathrm{v})$ P-teichoic acid in $0.15 \mathrm{M}-\mathrm{NaCl}+$ $0.15 \mathrm{M}$ citrate $\mathrm{pH} 7.4$ at $20^{\circ}$; the preparation was relatively monodisperse, the sedimentation coefficient $S$ of $9.5 \mathrm{~s}$ being indicative of a macromolecule.

After dialysis, the teichoic acids extracted by TCA were separated on columns of Sephadex $\mathrm{G} 75$ into two fractions having $K_{d}=0.0$ and 0.5 . The excluded fraction was also excluded from Sephadex G200 but included by $6 \%$ agarose. Agarose also gave a better separation of both teichoic acid fractions from contaminating nucleic acid

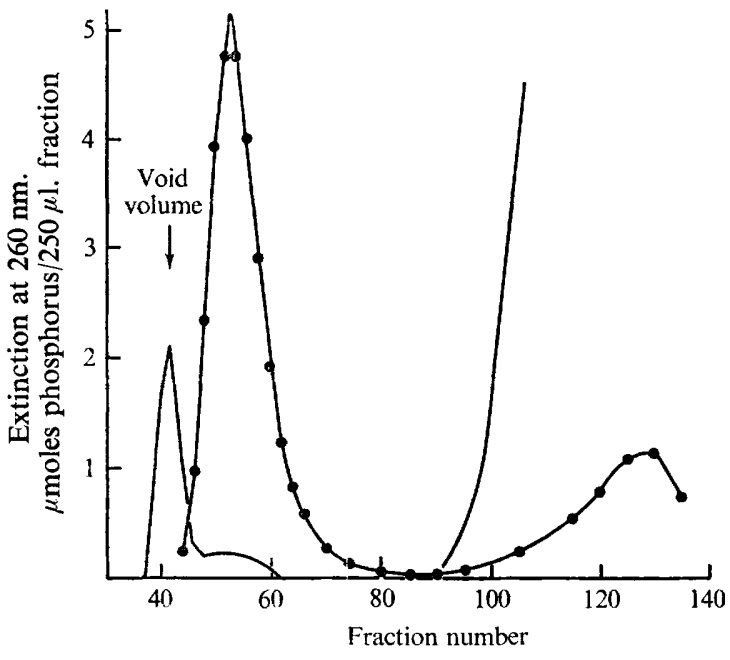

Fig. I

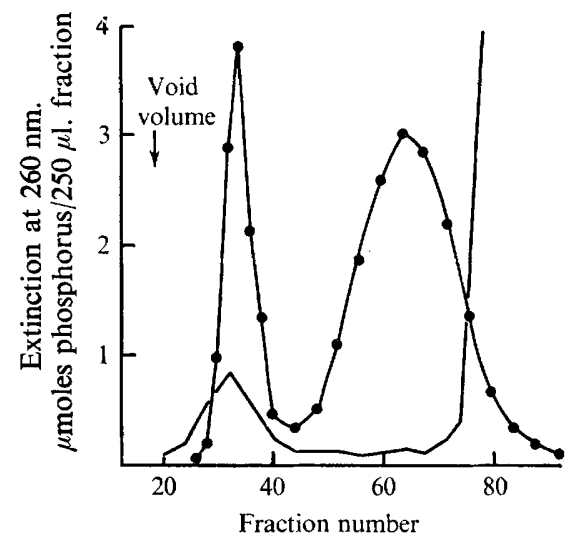

Fig. 2

Fig. I. Agarose gel $(6 \%)$ chromatography of teichoic acid obtained by phenol extraction of gel fraction from Lactobacillus fermenti. Column $(9 \mathrm{I} \times 2.6 \mathrm{~cm}$.) loaded with $360 \mathrm{mg}$. crude material and eluted with $0.2 \mathrm{~m}$-ammonium acetate, $\mathrm{pH} 6.9$, at a flow rate of $\mathrm{I} 3 \mathrm{ml} . / \mathrm{hr}$; $\mu$ moles phosphorus $/ 0.25 \mathrm{ml}$. fraction; continuous line, extinction at $260 \mathrm{~nm}$.

Fig. 2. Agarose gel $(6 \%)$ chromatography of teichoic acid obtained by TCA extraction of gel fraction from Lactobacillus fermenti. Column $(40 \times 2.6 \mathrm{~cm}$.) loaded with $270 \mathrm{mg}$. crude

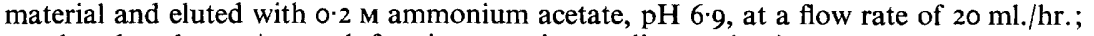
$\mu$ moles phosphorus $10.25 \mathrm{ml}$. fraction; continuous line, extinction at $260 \mathrm{~nm}$.

material and was used routinely in preference to Sephadex. The material with the higher apparent molecular weight was designated as HT-teichoic acid and the material with the lower apparent molecular weight as LT-teichoic acid, the notations (gel) and (sol) again, where necessary, being used to indicate origin. Fig. 2 shows the elution diagram of HT- and LT-teichoic acids (gel) from a column of $6 \%$ agarose. HT-and LT-teichoic acids (sol) showed similar elution patterns although the LT-fraction was contaminated with polysaccharide. Teichoic acids were recovered, after dialysis, by freeze drying.

\section{Composition of the teichoic acids}

Paper chromatography of acid hydrolysates of all teichoic acid preparations in solvents A and B showed glycerol, glyceromono- and di-phosphates, inorganic phosphate, glucose and galactose. D-Alanine was evident in HT and LT-teichoic acids and the corresponding amide was obtained from these preparations by ammonolysis. 
P-teichoic acids were devoid of all but traces of ester-linked alanine, as would be expected from the incubation at $\mathrm{pH} 8$ during the extraction procedure (see Methods), but showed a range of amino acids in acid hydrolysates indicative of the presence of a small amount of protein. Mole ratios of glucose: galactose and total sugar: phosphorus are shown in Table $\mathrm{r}$.

Table I. Mole ratios of glucose:galactose and total sugar:phosphorus in teichoic acid preparations

Fraction

P-Teichoic acid (gel)

P-Teichoic acid (sol)

HT-Teichoic acid (gel)

HT-Teichoic acid (sol)

LT-Teichoic acid (gel)

LT-Teichoic acid (sol)
Glucose: galactose

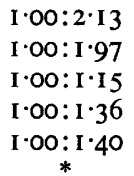

Total sugar:phosphorus

$$
\begin{gathered}
0.17: 1 \cdot 00 \\
0.18: 1 \cdot 00 \\
0.48: 1 \cdot 00 \\
0.50: 1 \cdot 00 \\
0.32: 1 \cdot 00 \\
*
\end{gathered}
$$

*Values for ratios not reported as analyses indicated that this fraction was contaminated with polysaccharide.

Table 2. Partial characterization of glycerol glycosides from alkaline hydrolysates of teichoic acid fractions

P-Teichoic acid

HT-Teichoic acid

LT-Teichoic acid

$R_{\text {glo }}$ (solvent B)

$R_{g l c}$ (solvent C)

Reaction with periodate

+ Schiff's reagent

Acid hydrolysis

Partial acid hydrolysis $\uparrow$

Periodate oxidation $\ddagger$

$\alpha$-Galactosidase action§
Glycoside $I$

+
+
-
0.46
0.39

Rapid

gal, glc*, glycerol gal, glucosyl-I-glycerol and traces of a disaccharide giving a yellow colour with the periodate+ Schiff's reagent

No resistant sugar, glycerol only product after reduction gal, glucosyl-1-glycerol
Glycoside 2

+
+
+
0.42
0.29

Slow gal, glc, glycerol
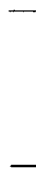

-

$*$ gal = galactose; glc $=$ glucose $. \quad \dagger 0.025 \mathrm{~N}-\mathrm{HCl}$ at $100^{\circ}$ for $5 \mathrm{hr} . \quad \ddagger$ Glycoside treated with $0.02 \mathrm{M}$-sodium metaperiodate for $72 \mathrm{hr}$, reduced with sodium borohydride and acid hydrolysed. $\S$ Almond emulsin (Sigma), which contains an $\alpha$-galactosidase.

All teichoic acid preparations were hydrolysed in alkali to a mixture of neutral components and organic phosphates. P- and HT-teichoic acids gave, in addition, a precipitate which could be extracted into acidified chloroform. Thin-layer silica gel chromatography of this material in solvent $\mathrm{E}$ gave a broad band $\left(R_{F} 0.5\right.$ to 0.6$)$ that reacted with iodine vapour and a specific fatty acid spray reagent. Examination of this fraction by gas-liquid chromatography indicated that palmitic acid was the major component. Quantitative estimation of fatty acid esters showed $3 \cdot 72 \%(w / w)$ and $5.10 \%(\mathrm{w} / \mathrm{w})$ respectively for P-teichoic acid (sol) and (gel) (as palmitic acid). A similar 
analysis of HT-teichoic acid was precluded by the presence of ester linked alanine. Fatty acids were not detected qualitatively as components of LT-teichoic acids.

Alkaline phosphornonoesterase treatment of alkali-hydrolysed teichoic acids gave a mixture of glycerol glycosides, glycerol, organic phosphate esters resistant to the enzyme and diglycerol monophosphate. The latter component was characterized by its chromatographic mobility and comparison with previously reported values, hydrolysis in alkali to glycerol and glycero-monophosphate, and rapid reaction with the periodate + Schiff's reagent on paper chromatograms. Characterization of the other phosphate esters will be reported elsewhere. The neutral fraction of glycerol glycosides was resolved into several components by preparative paper chromatography in solvent $\mathrm{C}$. The composition of these glycosides is shown in Table 2.

\section{Action of TCA on P-teichoic acid}

P-Teichoic acid (gel) (40 mg.) was dissolved in I0\% TCA (20 ml.) and kept at $4^{\circ}$ for $48 \mathrm{hr}$. Trichloracetic acid was removed by repeated extraction with ether and the

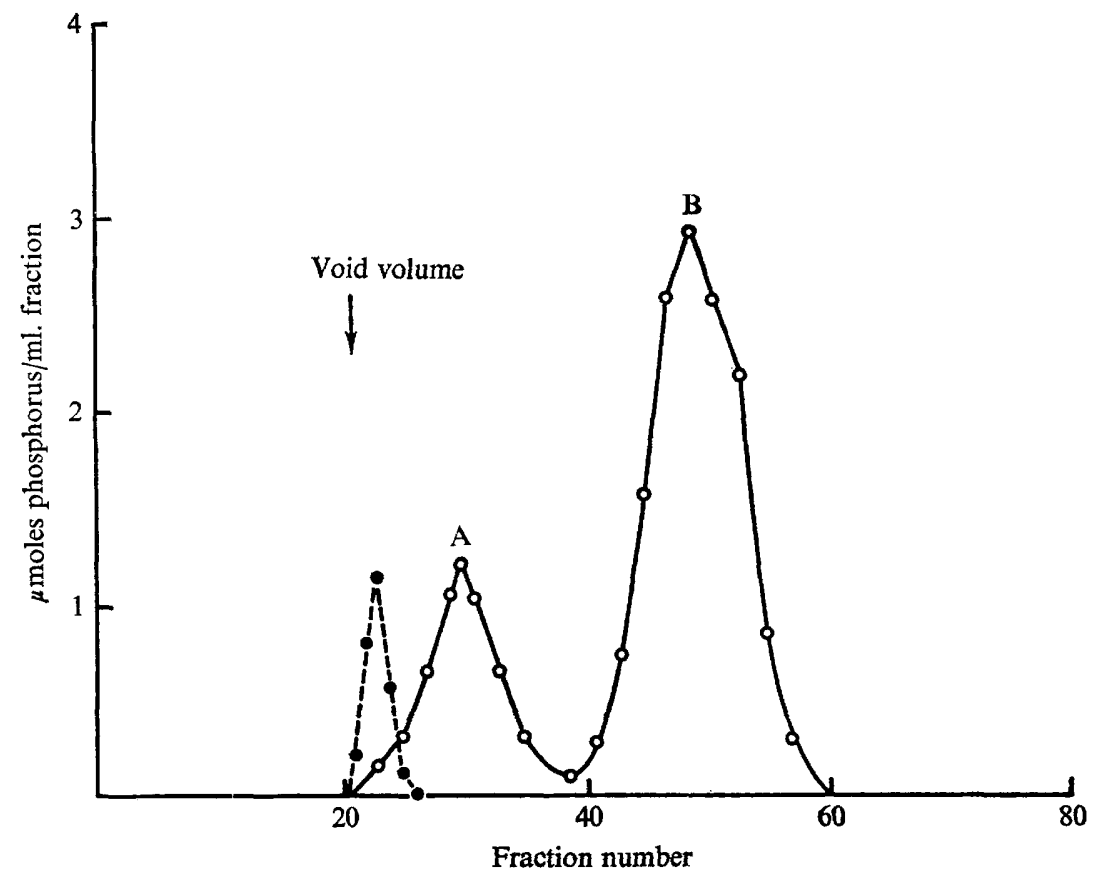

Fig. 3. Agarose gel ( $6 \%$ ) chromatography of P-teichoic acid (gel) from Lactobacillus fermenti after treatment with $10 \%$ TCA for $48 \mathrm{hr}$, column $(42 \times 2.6 \mathrm{~cm}$.) eluted with $0.2 \mathrm{M}$-ammonium acetate at a flow rate of $\mathrm{I} 5 \mathrm{ml}$./hr;, P-teichoic acid; $\bigcirc$, P-teichoic acid after TCA treatment,

aqueous layer, after neutralization with ammonia and concentration by rotary evaporation, was chromatographed in $0.2 \mathrm{M}$-ammonium acetate on a column of $6 \%$ agarose. The elution diagram is shown in Fig. 3. Two fractions of teichoic acid (A and B) corresponding in elution volume to HT- and LT-teichoic acids were obtained. Fraction A had a slightly greater elution volume than P-teichoic acid (gel) chromatographed on the same column. Alkaline hydrolysis of fraction A, like that of HT-teichoic acid, gave 
glycosides $I, 2$ and 3 (see Table 2) and fatty acids whereas fraction B showed only glycosides 2 and 3 after alkaline hydrolysis. Quantitative fatty acid ester analysis (as palmitic acid) gave $12.52(\mathrm{w} / \mathrm{w})$ and $0.5 \mathrm{I} \%(\mathrm{w} / \mathrm{w})$ for fractions $\mathrm{A}$ and $\mathrm{B}$ respectively.

The etheral extract of TCA degraded P-teichoic acid was washed with sodium bicarbonate solution to remove TCA and evaporated to dryness. Extraction of the residue with chloroform + methanol $(2: \mathrm{I}, \mathrm{v} / \mathrm{v})$ gave a small quantity of a component which had an $R_{F}$ of $0.9 \mathrm{I}$ on silica gel TLC-plates in solvent D. This material reacted with silver nitrate, iodine vapour, rhodamine $G$ and contained phosphorus; all these properties are indicative of a phospholipid.

\section{Major glycoside component of the glycolipid fraction}

Deacylation of the glycolipid fraction from a total lipid extract of Lactobacillus fermenti (see Methods) and chromatography in solvents $\mathrm{B}$ and $\mathrm{C}$ gave a major glycoside fraction identical to glycoside $I$ in chromatographic mobility, reaction with the periodate + Schiff's reagent, partial acid hydrolysis and treatment with $\alpha$-galactosidase (see Table 2).

\section{DISCUSSION}

It is evident from the gel chromatography of these teichoic acid preparations that phenol extraction gives a product of higher apparent molecular weight than the more commonly used method of cold TCA-extraction. Further, P-teichoic acid, and to a lesser extent HT-teichoic acid, could be obtained virtually free of nucleic acid material (Fig. I, 2), which suggests that sedimentation of teichoic acid with the ribosomal fraction at $100,000 \mathrm{~g}$ is not, as originally proposed (Critchley et al. 1962 ; Wicken \& Baddiley, 1963), a result of artefactual complex formation between RNA and teichoic acid. An alternative possibility, supported by the association of teichoic acid with membrane lipids (vide infra), is that the degree of disaggregation of membrane on disruption of organisms may determine whether or not the teichoic acid complex is sedimented at $100,000 \mathrm{~g}$ (Knox et al. 1970).

The products of alkaline and acidic hydrolysis of these teichoic acid preparations are typical of glycerophosphate polymers substituted with galactose and a disaccharide of galactose and glucose. The isolation of diglycerol monophosphate from phosphomonoesterase treated alkaline hydrolysates is indicative of the normal arrangement of phosphodiester groups between carbon atoms I and 3 of each glycerol moiety. Glycosides 2 and 3 (Table 2), from their slow reaction with the periodate + Schiff's reagent, are clearly 2-substituted glycerols and would be among the expected products from a similarly substituted teichoic acid hydrolysed in alkali and treated with phosphomonoesterase. P- and HT-teichoic acids showed, in addition, glycoside $I$ and fatty acid(s) as alkali degradation products. Glycoside $I$ has the properties (Table 2) of a galactosyl-glucosyl-I-glycerol. A rapid reaction of the glycoside with the periodate+ Schiff's reagent together with the production of glucosyl-I-glycerol by partial acid and by enzymic hydrolysis are indicative of substitution of a primary hydroxyl group of glycerol by the disaccharide galactosylglucose. The action of periodate, coupled with sodium borohydride reduction and acid hydrolysis, indicates either a $I \rightarrow 2$ or a $I \rightarrow 6$ linkage between the two sugar residues. The yellow colour produced by the periodate + Schiff's reagent acting on the disaccharide found in the partial acid hydrolysate of glycoside $I$ is consistent with a $I \rightarrow 2$ linkage (Wicken \& Baddiley, I963), and the action 
of $\alpha$-galactosidase shows this linkage to have the $\alpha$-configuration. Thus the evidence is consistent with glycoside $I$ being $O$ - $\alpha$-D-galactosyl-(I $\rightarrow 2$ )-D-glucosyl-(I $\rightarrow$ I)-glycerol. Further studies are in progress to confirm this tentative identification.

A I-substituted glycerol, such as glycoside $I$, could not be a constituent of a glycosidically substituted $x \rightarrow 3$ phosphodiester linked teichoic acid but could derive from a glycolipid (Brundish et al. 1965), the glycoside moiety of the glycolipid from Lactobacillus casei ATCC 7469 being identical with that proposed for glycoside $I$ (Shaw, Heatherington \& Baddiley, 1968). The glycolipid fraction of L. fermenti yielded a glycoside indistinguishable from glycoside $I$ suggesting that P-teichoic acid contains glycolipid.

In addition to glycolipid, tentative evidence has also been obtained for the presence of phospholipid as a component of P-teichoic acid. P-teichoic acid may therefore be regarded as a complex of teichoic acid with lipids and be referred to as lipoteichoic acid. Extraction with TCA results in the disruption of the complex with the formation of partially degraded lipoteichoic acid (HT-teichoic acid) and teichoic acid chains virtually devoid of lipid components (LT-teichoic acid).

The apparent large molecular size of lipoteichoic acids might be explained by micelle formation. The water solubility of P-teichoic acids and the resistance of the lipid moiety to extraction by chloroform-methanol (see Methods) is indicative of an amphipathic structure in which the hydrophobic lipid portion of the complex is 'buried' as well as firmly bound to the hydrophilic teichoic acid chains. Such micellar formations have been reported for lipopolysaccharides and phospholipids from Gramnegative organisms (Rothfield \& Horne, 1967). The presence of teichoic acid as a lipid complex is also consistent with its being present as an integral component, with phospholipid, of the common leaflet structure of the cell membrane. In Gramnegative organisms, which lack teichoic acid, it has been proposed that lipopolysaccharide fulfils a similar role in the outer cell envelope (Rothfield \& Horne, 1967).

This work was supported by a grant (to K. W. K.) from the National Health and Medical Research Council of Australia.

\section{REFERENCES}

Ames, B. N. (1966). Assiays of phosphate and phosphatases. Methods in Enzymology 8, I 5.

Archibald, A. R., Baddiley, J. \& Blumsom, N. (I968). The teichoic acids. Advances in Enzymology 30, 223.

Baddiley, J., Buchanan, J. G., Handschumacher, R. E. \& Prescott, J. F. (1956). Biosynthesis of purine nucleotides. I. Preparation of N-glycylglycosylamines. Journal of the Chemical Society $\mathrm{p}$. 2818.

BRundish, D. E., Shaw, N. \& BADDILEY, J. (I965). The occurrence of glycolipids in Gram-positive bacteria. Biochemical Journal 94, 2 I $c$.

Burger, M. M. (1966). Teichoic acids: Antigenic determinants, chain separation and their location in the cell wall. Proceedings of the National Academy of Sciences of the United States of America 56, 910.

Burger, M. M. \& Glaser, L. (1964). The synthesis of teichoic acids. I. Polyglycerophosphate. Journal of Biological Chemistry 239, 3168.

Critchley, P., ARChibald, A. R. \& Baddiley, J. (1962). The intracellular teichoic acid from Lactobacillus arabinosus, I7-5. Biochemical Journal 85, 420.

CONSDEN, R. \& GoRdon, A. H. (1948). Effect of salt on partition chromatograms. Nature, London I62, 180 . 
DitTmer, J. C. \& Lester, R. L. (1964). A simple, specific spray for the detection of phospholipids on thin-layer chromatograms. Journal of Lipid Research $5,126$.

DudZINSKI, A. E. (1967). A spray sequence for the detection of free fatty acids. Journal of Chromatography 31, 560 .

Forrester, I. T. \& WiCKEN, A. J. (I966). The chemical composition of the cell walls of some thermophilic bacilli. Journal of General Microbiology 42, 147.

Hay, J. B., Wicken, A. J. \& BadDiley, J. (1963). The location of intracellular teichoic acids. Biochimica et Biophysica Acta 71, I88.

Hanes, C. S. \& Isherwood, F. A. (1949). Separation of phosphoric esters on the filter paper chromatogram. Nature, London 164, 1107.

HewetT, M. J., Knox, K. W. \& WiCken, A. J. (1970). Studies on the group F antigen of lactobacilli: Detection of antibodies by haemagglutination. Journal of General Microbiology 60, 3 I 5 .

Hugget, A. St G. \& Nixon, D. A. (I957). Use of glucose oxidase, peroxidase and $o$-dianisidine in determination of blood and urinary glucose. Lancet 273,368 .

Jeanes, A., Wise, C. S. \& Dimler, R. J. (I95I). Improved techniques in paper chromatography of carbohydrates. Analytical Chemistry 23, $4 \mathrm{I} 5$.

Knox, K. W., Hewett, M. J. \& WICKEN, A. J. ( I970). Studies on the group F antigen of lactobacilli: Antigenicity and serological specificity of teichoic acid preparations. Journal of General Microbiology 60, 303 .

KNox, K. W. \& Holmwood, K. J. (I968). Structure of the cell wall of lactobacilli. Role of muramic acid phosphate in Lactobacillus fermenti. Biochemical Journal ro8, 363.

ROTHFIELD, L. \& HoRNE, R. W. (I967). Reassociation of purified lipopolysaccharide and phospholipid of the bacterial cell envelope: Electron microscopic and monolayer studies. Journal of Bacteriology 93, I705.

SASTRY, P. S. \& KATES, M. (1964). Lipid components of leaves. v. Galactolipids, cerebrosides, and lecithin of runner bean leaves. Biochemistry 3, 127 I.

ShARPE, M. E. (I955). A serological classification of Lactobacilli. Journal of General Microbiology I2, 107.

Sharpe, M. E., Davison, A. L. \& Baddiley, J. (1964). Teichoic acids and group antigens in lactobacilli. Journal of General Microbiology 34, 333.

SharPe, M. E. \& WheATER, D. M. (1957). Lactobacillus helveticus. Journal of General Microbiology I6, 676 .

Shatтock, P. M. F. \& Sмiтh, D. G. (1963). The location of the group D antigen in a strain of Streptococcus faecalis var. liquifaciens. Journal of General Microbiology 31, iv.

Shaw, N., Heatherington, K. \& Baddiley, J. (I968). The glycolipids of Lactobacillus casei atcC 7469. Biochemical Journal Io7, 491.

Shockman, G. D. \& Slade, H. D. (I964). The cellular location of the streptococcal group D antigen. Journal of General Microbiology 37, 297.

Smith, D. G. \& Shatтock, P. M. F. (1964). The cellular location of antigens in streptococci of groups $\mathrm{D}, \mathrm{N}$ and Q. Journal of General Microbiology 34, 165.

SNYDER, F. \& STEPHENS, N. (1959). A simplified spectrophotometric determination of ester groups in lipids. Biochimica et Biophysica Acta 34, 244.

Trevelyan, W. E., Procter, D. P. \& Harrison, J. S. (1950). Detection of sugars on paper chromatograms. Nature, London 166, 444.

Wells, M. A. \& Dittmer, J. C. (1963). The use of Sephadex for the removal of non-lipid contaminants from lipid extracts. Biochemistry 2, I 259.

WiCKEN, A. J. (1966). The glycerol teichoic acid from the cell wall of Bacillus stearothermophilus B 65. Biochemical Journal 99, 108.

WiCkEN, A. J. \& BADDILEY, J. (I963). Structure of intracellular teichoic acids from group D streptococci. Biochemical Journal 87, 54.

Wilkinson, S. G. (1968). Glycosyl diglycerides from Pseudomonas rubescens. Biochimica et Biophysica Acta 164, 148.

Young, F. E. \& JACKSON, A. P. (I966). Extent and significance of contamination of DNA by teichoic acid in Bacillus subtilis. Biochemical and Biophysical Research Communications 23, 4. 